\title{
Comparación de la percepción subjetiva del esfuerzo entre partidos amistosos y diferentes tipos de sesión en futbolistas profesionales \\ Comparison of perceived exertion in friendly matches and different types of training sessions in professional soccer players
}

\author{
*Miguel Ángel Campos Vázquez, **Francisco JavierToscano Bendala \\ *Universidad Pablo de Olavide (España), **Universidad Católica San Antonio (España)
}

\begin{abstract}
Resumen. El objetivo de la investigación fue comparar la percepción subjetiva del esfuerzo (RPE) y la carga derivada de ella (RPE-TL) durante partidos amistosos (PA) con una participación de 45 minutos por jugador (PA.45) o superior a 65 minutos (PA+65), y diferentes tipos de sesión de entrenamiento: condición física (CF), táctica (TAC) y activación pre-partido (ACTV), en jugadores profesionales de fútbol. 12 futbolistas pertenecientes al mismo equipo ( $1^{\text {a }}$ división española) participaron en este estudio. La RPE fue registrada tras cada sesión de entrenamiento y PA, cuantificándose posteriormente la carga interna derivada de ella (RPE-TL). Los resultados reflejaron que los PA+65, tuvieron una RPE substancialmente mayor que los PA.45 (7.8 vs 6.8). Además, todos los tipos de sesión reflejaron una RPE y RPE-TL substancialmente inferior a los PA+65. Tan solo las sesiones de CF alcanzaron valores de RPE y RPE-TL superiores a los reflejados en los PA.45 (7.1 vs. 6.8 y 597 vs. 509 unidades arbitrarias respectivamente). Estos resultados nos muestran que las demandas perceptuales de la competición no fueron replicadas en los diferentes tipos de sesiones de entrenamiento analizadas. Por tanto, podría ser necesario aumentar el volumen y/o la exigencia de algunas sesiones de entrenamiento, para someter a los jugadores a una exigencia similar a la de la competición.
\end{abstract}

Palabras clave: Percepción subjetiva esfuerzo, Carga de entrenamiento, Partidos amistosos, Sesiones entrenamiento, Fútbol.

Abstract. The aim of the study was to compare perception of exertion (RPE) and RPE-derived internal training load between friendly matches (FM) played during 45 minutes (FM.45) or more than 65 minutes (FM+65), and different types of training sessions: fitness (FIT), tactical (TAC) and prematch activation (ACTV) in professional soccer players. 12 soccer players from the same team (1 ${ }^{\text {st }}$ Spanish Division) participated in this study. RPE was registered after every training session and FM. Afterward, RPE-derived internal load was calculated (RPE-TL). Results showed that FM+65 obtained a substantially higher RPE than FM.45 (7.8 vs 6.8). Besides, all types of training sessions reflected a substantially lower RPE and RPE-TL than FM+65. Only FIT sessions reached RPE and RPE-TL values higher than those reflected in FM.45 (7.1 vs. 6.8 and 597 vs. 509 arbitrary units respectively). These results show that perceptual demands of competition were not replicated in the different types of training sessions analysed. It may be necessary to increase the volume and/or the exertion of certain training sessions to bring players closer to the demands of competition. Key Words: perceived exertion, Training load, Friendly matches, Training sessions, Soccer.

\section{Introducción}

En fútbol, diferentes tipos de sesión son habitualmente realizados durante cada microciclo de entrenamiento (Campos-Vazquez, MendezVillanueva, Gonzalez-Jurado, Leon-Prados, Santalla \& Suarez-Arrones, 2015; Jeong, Reilly, Morton, Bae \& Drust, 2011), con el objetivo de que los jugadores mejoren las habilidades técnico-tácticas necesarias para el juego y para alcanzar un nivel de condición física que les permita soportar las intensificadas demandas de la competición. Estas sesiones, suelen estar distribuidas dentro cada microciclo teniendo en cuenta su objetivo, contenido y carga demandada, para que el jugador llegue al partido en unas óptimas condiciones físicas, ya que el partido de competición es el estímulo más importante de la planificación semanal de los futbolistas (Arcos, Yanci, Mendiguchia \& Gorostiaga, 2014; Impellizzeri, Rampinini, Coutts, Sassi \& Marcora, 2004).

Una de las inquietudes en el ámbito del entrenamiento es conocer si se replican durante las sesiones de entrenamiento las demandas de la competición. Para ello, es necesario monitorizar de forma habitual tanto las demandas físicas como fisiológicas de las sesiones de entrenamiento y los partidos de competición. En este contexto, debería ser una prioridad conocer la carga óptima en la que los procesos de adaptación se suceden, tomando como referencia las demandas de la competición (Owen, Djaoui, Newton, Malone \& Mendes, 2017). Esta información, puede ser usada en el diseño de las sesiones de entrenamiento, con el objetivo de aproximar a los jugadores a los valores de carga e intensidad alcanzados en los partidos, ya que el rendimiento podría mejorar más cuando los estímulos de entrenamiento simulan las demandas fisiológicas y los patrones de movimiento de los partidos de competición (Sampaio, Abrantes \& Leite, 2009).

En la actualidad son diversas las propuestas de monitorización de la carga de entrenamiento empleadas por los preparadores físicos de

Fecha recepción: 30-01-17. Fecha de aceptación: 02-10-17 Miguel Ángel Campos Váquez macamvaz@upo.es fútbol, durante las sesiones de entrenamiento y los partidos de competición. En las sesiones de entrenamiento, es habitual valorar la carga interna de entrenamiento (estrés fisiológico) a través del análisis de la frecuencia cardíaca (FC) (Campos-Vazquez et al., 2015; Dellal et al., 2012; Jeong et al., 2011) o la percepción subjetiva del esfuerzo (RPE) (Arcos et al., 2014; Campos-Vazquez et al., 2015; Impellizzeri et al., 2004). Recientes investigaciones, han mostrado como existen diferencias entre la carga diaria (FC y RPE) de las sesiones habitualmente planteadas por los entrenadores en equipos profesionales. Así, Jeong et al. (2011), concluyeron que las sesiones de condición física, reflejaban unos valores de intensidad superiores a los de las sesiones técnicotácticas (RPE: 7 vs 4 u.a). Estos resultados están en consonancia con los mostrados por Campos-Vazquez et al. (2015), con valores de RPE para las sesiones de condición física (7.4 unidades arbitrarias, u.a.) superiores a las sesiones técnico-tácticas (4.8 u.a.) y las sesiones de activación previas a la disputa de los partidos de competición (4.5 u.a.). Sin embargo, en ninguno de los mencionados estudios, se obtuvieron datos derivados de los partidos de competición.

Por otro lado, la carga externa (estímulos físicos) suele ser normalmente monitorizada por medio de dispositivos GPS (Anderson, Orme, Di Michele, Close, Morgans, Drust \& Morton, 2016; Malone, Di Michele, Morgans, Burgess, Morton \& Drust, 2015). Estos dispositivos, proporcionan información de determinadas variables como la distancia total, la distancia cubierta en diferentes zonas de velocidad, los impactos o el número de aceleraciones y deceleraciones (Cummins, Orr \& O’Connor, 2013). Habitualmente dichas variables se presentan en las investigaciones de forma aislada, y tan sólo recientemente se ha intentado diseñar un modelo de análisis de carga externa en el que los valores de los entrenamientos se expresan en relación a los alcanzados en la competición (Owen et al., 2017). Sin embargo, una dificultad para llevar a cabo este planteamiento, surge del hecho de que en competición, se monitoriza normalmente la carga, a través de sistemas de video-análisis computerizados semi-automáticos (Amisco, Prozone)(Di Salvo, Baron, Tschan, Calderon Montero, Bachl \& Pigozzi, 2007; Di Salvo, Gregson, Atkinson, Tordoff \& Drust, 2009; Rampinini, Coutts, Castagna, Sassi 
\& Impellizzeri, 2007), ya que la utilización tanto de pulsómetros como de dispositivos GPS ha estado prohibida en los partidos oficiales de competición hasta el comienzo de la temporada 2015-16. Además, pese a que en la actualidad está permitido su uso, son muchos los futbolistas profesionales que no se encuentran cómodos durante el partido teniendo que llevar petos para GPS o cintas de monitorización de frecuencia cardíaca, resultando de esta forma complicado monitorizar con estos dispositivos a todos los jugadores, durante los partidos de competición. Por tanto, y debido a la diversidad de métodos y herramientas de monitorización utilizadas en entrenamientos y competición, resulta en ocasiones muy difícil poder comparar la intensidad y carga derivada de los entrenamientos con la de la competición.

La RPE, valorada mediante una escala de Borg-10 (Borg, Hassmen \& Lagerstrom, 1987), es una herramienta simple, no invasiva, barata y válida para valorar la intensidad del ejercicio físico en fútbol (Los Arcos, Mendez-Villanueva, Yanci \& Martinez-Santos, 2016) y otros deportes colectivos (Clarke, Farthing, Norris, Arnold \& Lanovaz, 2013; Murillo, Alvarez \& Manomelles, 2016). Por ello, la RPE ha sido habitualmente utilizada en investigación para valorar la intensidad de tareas como las situaciones reducidas de juego (Dellal, Drust \& Lago-Penas, 2012), sesiones de entrenamiento, (Campos-Vazquez et al., 2015), o partidos de competición (Los Arcos et al., 2016). Además, a través de ella, se puede estimar la carga interna de entrenamiento (RPE-TL) siguiendo la propuesta de Foster et al. (2001), al multiplicar la RPE por el volumen total (minutos) de la sesión. Esta propuesta ha alcanzado relaciones significativas con otros métodos basados en el análisis de la FC, como TRIMP de Banister, o las propuestas de Edwards o Stagno (Alexiou \& Coutts, 2008; Campos-Vazquez et al., 2015; Impellizzeri et al., 2004). Puesto que se trata de una herramienta no invasiva y fácil de implementar, este método de monitorización puede utilizarse tanto en sesiones de entrenamiento como en partidos de competición, permitiendo una comparativa fiable de los valores derivados de ambas.

Por tanto, el principal objetivo de la investigación fue comparar la percepción subjetiva del esfuerzo proporcionada por los jugadores así como la carga derivada de ella, durante partidos amistosos y diferentes tipos de sesión en futbolistas profesionales.

\section{Material y Método}

\section{Participantes}

Un total de 22 jugadores profesionales de fútbol participaron inicialmente en este estudio (edad: $28.1 \pm 4.7$ años; altura: $179.7 \pm 4.1 \mathrm{~cm}$; peso: $78.2 \pm 7.2 \mathrm{Kg}$; sumatorio seis pliegues: $51.0 \pm 8.6$; \% grasa(Faulkner): 12.1 \pm 1.6 ). Sin embargo, la muestra quedó reducida a 12 jugadores (cinco defensas, cuatro centrocampistas y tres delanteros) ya que 10 jugadores no tuvieron una participaron suficiente (al menos dos partidos) en los partidos amistosos, condición necesaria para poder efectuar las comparaciones con los diferentes tipos de sesiones. Todos los jugadores pertenecían al mismo equipo, que competía en la Liga BBVA ( $1^{\text {a División }}$ de la Liga Española, temporada 2015-16). Todos los sujetos fueron informados previamente sobre el objetivo del estudio y tipo de pruebas a las que se sometería, y dieron su consentimiento informado siguiendo las indicaciones de la Declaración de Helsinki.

\section{Procedimiento}

El estudio se llevó a cabo durante una pretemporada completa y tuvo una duración de siete semanas (Julio-Agosto 2015). Durante ese tiempo los jugadores realizaron entre cinco y nueve sesiones de entrenamiento (ENT) y jugaron entre uno y dos partidos amistosos (PA) cada semana. Las ENT tuvieron una duración media de $\sim 75$ minutos (con un rango de entre 60 y $90 \mathrm{~min}$ ) y fueron agrupadas en diferentes tipologías atendiendo al objetivo, distancia al partido de competición y contenidos desarrollados en las mismas, definiéndose de esta forma los siguientes tipos de ENT:

- Sesiones Tácticas (TAC). Estas sesiones de iniciaban con un calentamiento técnico (15 minutos) y posteriormente se realizaba una tarea de activación jugada en espacio reducido (15 minutos: rondo-juego de posesión). Tras esta primera parte, se realizaban tareas condicionadas de 11 vs. 11 (45-60 minutos) para la mejora del modelo del juego del equipo en espacios moderadamente amplios ( 70 x 65 m, largo y ancho respectivamente).

- Sesiones Condición Física(CF). Estas sesiones solían comenzar con entrenamiento de fuerza general (ejercicios como sentadillas, arrastres con trineo y cambios de dirección con sobrecargas) o funcional (ejercicios de refuerzo muscular con elásticos, CORE y ejercicios excéntricos de isquiotibiales y adductores), llevado a cabo en el terreno de juego, durante un tiempo de 30 minutos aproximadamente. Posteriormente se realizaban tareas de acondicionamiento físico tales como interval training de alta intensidad, circuitos físico-técnicos (sucesión de esfuerzos explosivos tales como sprint, saltos, cambios de dirección, etc. y acciones técnicas tales como pases, conducción o finalización) y/o situaciones reducidas de juego ( 4 vs 4 / 6 vs 6 ) con porterías y porteros. Esta segunda parte de la sesión se prolongaba por un tiempo de unos 45 minutos.

- Sesiones Activación Pre-Partido (ACTV). Estas sesiones se realizaban el día previo a la disputa de un partido amistoso. Tras el calentamiento se realizaban acciones de finalización a la máxima velocidad. Posteriormente los jugadores realizaban entre 15-20 minutos de partido 10 vs. 10 en campo de 70 x 65 m, para terminar la sesión de entrenamiento con acciones a balón parado.

Además, durante la pretemporada se jugaron PA, que fueron clasificados en dos categorías en función de la participación de los jugadores en los mismos:

- PA. 45. La participación del jugador en el partido fue de 45 minutos (un tiempo completo).

- PA+65. La participación del jugador en el partido fue superior a 65 minutos por jugador (primer tiempo completo y al menos 20 minutos del segundo tiempo).

El sistema de juego empleado por el equipo en todos los PA monitorizados fue 1-5-3-2, es decir, un portero, cinco defensas, cuatro medio y dos delanteros.

El tiempo total de cada ENT y PA fue registrado para cada jugador (incluyendo calentamiento y pausas, pero no el descanso en el caso de los PA+65). Los registros individuales de los jugadores que no completaron algún ENT (por lesión o reducción en la carga de entrenamiento) fueron excluidos del análisis. La duración media, el número de sesiones monitorizadas y el número de registros individuales para cada tipo de ENT y PA pueden verse en la Tabla 1. Un total de 379 registros individuales cumplieron todos los requisitos y fueron incluidos en el análisis.

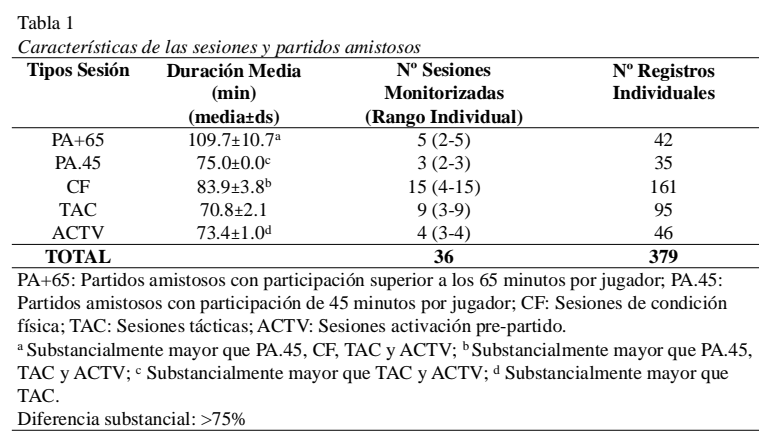

Se registró la RPE diariamente. Para ello, 30 minutos después de concluir cada ENT o PA, los jugadores proporcionaban el valor de RPE (unidades arbitrarias, u.a.) usando una escala de Borg-10 (Borg et al., 1987). Así, el preparador físico preguntaba a cada jugador en privado (sin la presencia de otros compañeros, y sin poder ver los valores marcados por el resto de compañeros), cómo de exigente había resultado el ENT/PApara él. Posteriormente, el jugador, seleccionaba un valor entre 0 y 10 (con incrementos de 0.5 ). Todos los jugadores estaban previamente familiarizados con el uso de la escala, al utilizarla diariamente tanto en entrenamientos como en la competición, durante la temporada anterior, y en la semana previa al comienzo de la investigación. 
Posteriormente se cuantificó la carga interna de entrenamiento derivada de la RPE (RPE-TL), realizando el cálculo propuesto por Foster et al. (2001), multiplicándose el valor de RPE proporcionado por el futbolista por la duración total del entrenamiento (min), y siendo también valorada en unidades arbitrarias (u.a.). Esta propuesta ha mostrado ser una forma válida de control de la carga interna, al alcanzar elevadas correlaciones con diversos métodos basados en el análisis de la frecuencia cardíaca, tales como TRIMP de Edwards o TRIMP modificados de Stagno (Impellizzeri et al., 2004; Campos-Vazquez et al., 2015).

\section{Análisis Estadístico}

Los resultados son expresados como media \pm desviación estándar (DE). Los datos fueron evaluados para la significación práctica utilizando inferencias basadas en la magnitud de los cambios (Hopkins, 2006). El análisis de los datos consistió en comparar la magnitud de los cambios en el volumen, la RPE y la carga derivada de la RPE entre los partidos amistosos disputados y las distintas sesiones de entrenamientos.

Estos análisis se llevaron a cabo utilizando una hoja de cálculo estadístico modificado (Hopkins, 2006). La hoja calcula diferencias entre grupos o tamaños de efecto estandarizados (TE, 90\% limites confianza [CL]), utilizando la desviación estándar combinada. Los criterios para interpretar la magnitud de los TE fueron; 0.0 a 0.2 trivial, 0.2 0.6 pequeño, 0.6-1.2 moderado, 1.2-2.0 grande $\mathrm{y}>2.0$ muy grande (Batterham y Hopkins, 2006).

Los descriptores cualitativos propuestos por Batterham \& Hopkins (2006) se utilizaron para interpretar las probabilidades de que el efecto verdadero puede ser perjudicial, trivial o beneficioso: $<1 \%$, casi seguro que no (CSN); El 1-4\%, muy improbable (MI); 5- 24\%, improbable (IM); 25-74\%, posible (POS); 75 a 94\%, probable (PRO); 95-99\%, muy probable (MPR); > 99\%, casi seguro (CS). Una diferencia substancial fue establecida como >75\% (Batterham \& Hopkins, 2006).

\section{Resultados}

Los PA+65 tuvieron una RPE substancialmente mayor a la de los PA.45(TE: 1.4, grande, CS) y a la de las sesiones CF (TE: 0.7 , moderado, MPR), TAC (TE: 2, muy grande, CS) y ACTV (TE: 2.9, muy grande, CS) (Figura 1). Los PA.45 tuvieron una RPE substancialmente menor a las sesiones CF (TE: 0.4, pequeño, PRO), pero superior a las sesiones TAC (TE: 0.4 , pequeño, PRO) y ACTV (TE: 1.2 , grande, CS) (Figura 1). Las sesiones de CF, mostraron una RPE substancialmente superior a las sesiones TAC (TE: 2.2, muy grande CS) y ACTV (TE: 4 , muy grande, CS). Finalmente, las sesiones TAC reflejan un RPE substancialmente mayor que las sesiones ACTV (TE: 1.1, moderado, CS).

Los PA+65 tuvieron una RPE-TL substancialmente mayor a la de los PA.45 (TE: 2.9, muy grande, CS) y a la de las sesiones CF (TE: 1.8, grande, CS), TAC (TE: 3.5 muy grande, CS) y ACTV (TE: 4, muy grande, CS). (Figura 2). Los PA.45 tuvieron una RPE-TL superior a las sesiones TAC (TE: 0.8 , moderado, MPR) y ACTV (TE: 1.3 moderado, CS), pero inferior a las sesiones CF (TE: 1.0, moderado, MPR). La carga perceptiva en las sesiones de CF fue substancialmente mayor que en las sesiones TAC (TE: 6.2 muy grande, CS) y en las sesiones ACTV (TE: 8 muy grande, CS). Finalmente la RPE-TL en las sesiones TAC, fue substancialmente mayor que en las sesiones ACTV (TE: 1.1 moderado, MPR). La duración media de los partidos y los diferentes tipos de sesión pueden verse en la Tabla 1 .

\section{Discusión}

El principal objetivo de la investigación fue comparar la RPE y la carga derivada de ella (RPE-TL) durante partidos amistosos y diferentes tipos de sesión de entrenamiento en futbolistas profesionales. Los datos reportados en nuestro estudio, muestran como ningún tipo de sesión alcanzó los valores de intensidad y carga perceptiva de los PA+65. Además, los PA+65, alcanzaron unos valores de RPE substancialmente superiores a los PA.45, reflejando que el valor de RPE está claramente condicionado por el tiempo de exposición, no debiendo ser por tanto catalogado como un valor de intensidad «puro».

Un objetivo prioritario del entrenamiento debe ser preparar a los jugadores para el esfuerzo y exigencia físico-fisiológica de la competición. Así, tanto entrenadores como preparadores físicos y especialistas en acondicionamiento físico, deberían elaborar estrategias de entrenamiento adecuadas (diseñar tareas / sesiones de entrenamiento) para acercar a los jugadores a las demandas competitivas (Scott, Lockie, Davies, Clark, Lynch \& de Jonge, 2014). De esta forma, los valores de carga e intensidad alcanzados por los jugadores durante los partidos de competición, podrían considerarse como una referencia válida e interesante para el diseño, planificación y periodización de las sesiones de entrenamiento.

Los valores de RPE promediados en nuestro estudio en los PA+65 (7.8 \pm 0.9 u.a) fueron aparentemente inferiores a los promediados en una investigación previa realizada con jóvenes futbolistas de entre 1418 años (8.4-8.5 u.a) (Wrigley, Drust, Stratton, Scott \& Gregson, 2012). Estas diferencias podrían ser debidas a que en nuestro estudio, no todos los jugadores evaluados alcanzaron la máxima participación de minutos en el partido, como sí sucedió en el estudio de Wrigley et al. (2012). El criterio que incluimos para valorar la RPE de los PA+65 en nuestra investigación, fue que los jugadores tuvieran una participación mínima de 65 minutos de juego (45 minutos de la primera parte y al menos 20 de la segunda parte). Por otra parte, el valor promediado por nuestros jugadores durante los PA.45 fue de $6.8 \pm 1.0$ u.a., substancialmente inferior al de los $\mathrm{PA}+65$. Estos resultados, parecen estar en consonancia con los reportados por diversos estudios realizados con jóvenes futbolistas profesionales (Arcos et al., 2014; Los Arcos et al., 2016), que muestran como los valores de RPE de los jugadores en la competición, parecen estar condicionados por el tiempo de participación de los

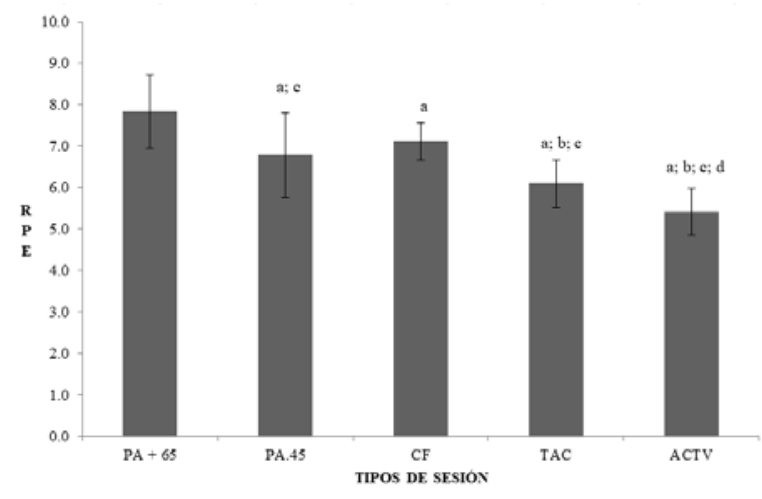

Figura 1. Medias de RPE (percepción subjetiva del esfuerzo) en partidos amistosos y distintos tipos de sesiones de entrenamiento. a. Substancialmente menor que PA+65 (partidos amistosos

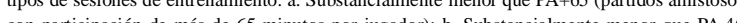
con participación de más de 65 minútos por jugador), b. Substanciante menor que PA.45 (partidos amistosos con paticipacion de 45 minutos por jugador), c. Substancialmente meno que CF (sesiones de condición fisica). d. Substancialmente menor que TAC (sesiones tácticas). ACTV (Sesiones activación). Diferencia substancial: $>75 \%$

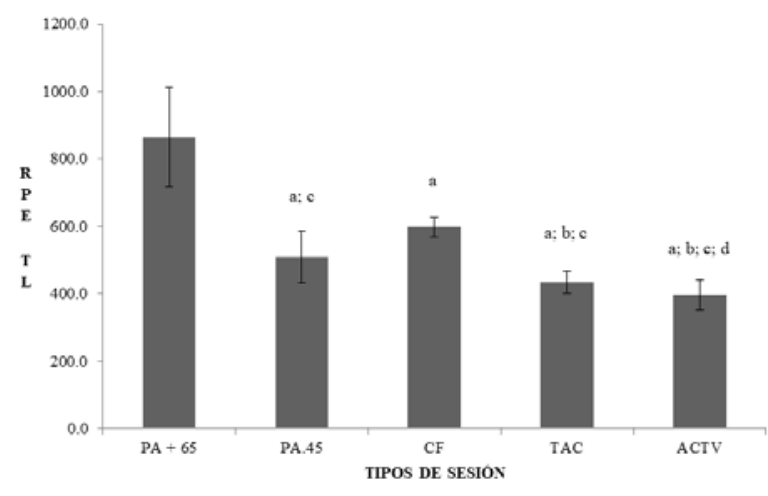

Figura 2. Medias de carga interna (RPE- TL) en partidos amistosos y distintos tipos de sesiones de entrenamiento. a. Substancialmente menor que PA+65 (partidos amistosos con participación de entés 65 . a. Substa ju

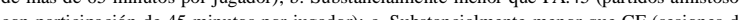
con participación de 45 minutos por jugador); c. Substancialnente menor que CF (sesiones de condición física). d. Substancialmente menor que TAC (sesiones tácticas). ACTV (sesiones activación). Diferencia substancial: $>75 \%$ 
futbolistas en el partido, con valores de RPE de 7.4 y 6.4 (RPE muscular y RPE respiratorio respectivamente) para aquellos con una participación superior a 70 minutos (Arcos et al., 2014).

Investigaciones previas, compararon la carga perceptual (Los Arcos, Gil-Rey, Izcue \& Yanci, 2013) o la intensidad y carga perceptual (Campos-Vazquez et al., 2015; Jeong et al., 2011) en diferentes tipos de sesión, aunque sin compararlos con la competición. Los Arcos et al. (2013), clasificaron las sesiones realizadas por jóvenes jugadores profesionales, en 4 categorías de acuerdo a su contenido y ubicación en la estructura semanal: resistencia + técnico-táctico; fuerza + técnico-táctico; técnico-táctico y táctico-estratégico, encontrando que las sesiones con mayor carga perceptiva fueron las realizadas en la parte central del microciclo semanal (fuerza + técnico-táctico). Dichas sesiones, obtuvieron valores cercanos a las 300 u.a., claramente inferiores a las sesiones con menor carga de las analizadas en nuestro estudio (ACTV: 395.8 \pm 44.5 u.a.). Estos resultados podrían reflejar las diferencias entre la carga soportada por los jugadores profesionales jóvenes ( $2^{\mathrm{a}}$ División $\mathrm{B}$, liga española) y los profesionales de primer nivel ( $1^{\text {a }}$ División, liga española).

A pesar de que ningún tipo de las sesiones incluidas en nuestro estudio, consiguió alcanzar los valores de carga e intensidad perceptiva de los PA+65, las sesiones de CF fueron las únicas con valores de RPE y RPE-TL superiores a los reflejados en los PA.45 (7.1 vs. 6.8 y 597 vs. 509 u.a. respectivamente). Los valores reportados en nuestras sesiones CF, están en consonancia con los mostrados para este tipo de sesiones en una investigación previa realizada con futbolistas profesionales de la liga española (7.4-6.9 u.a y 517-642 u.a. para RPE y RPE-TL respectivamente) (Campos-Vazquez et al., 2015). Estas sesiones, que fueron las de mayor duración de las analizadas (Tabla 1), están diseñadas para intentar mejorar el rendimiento físico de los jugadores, preparándolos para las exigencias físicas y fisiológicas de los partidos de competición (Jeong et al., 2011; Wrigley et al., 2012), a través de ejercicios como circuitos físico-técnicos, situaciones reducidas de juego o entrenamiento interválico de alta intensidad. Parece pues evidente, que los entrenadores utilizan estas sesiones como el estímulo de entrenamiento con más carga y volumen del microciclo (obviando el partido de competición) tanto en pretemporada (Jeong et al., 2011) como en la temporada competitiva (Campos-Vazquez et al., 2015; Jeong et al., 2011; Los Arcos et al., 2013), hecho que explicaría los superiores valores encontrados en CF respecto a PA.45.

A pesar de que las sesiones TAC utilizan tareas de entrenamiento (11 vs. 11 en campo moderadamente amplio) mucho más cercanas al juego real, reflejaron valores de carga e intensidad perceptiva substancialmente inferiores a las dos modalidades de partido analizadas, y a las sesiones de CF (Figuras 1 y 2). Los entrenadores habitualmente utilizan estas sesiones para mejorar el modelo de juego del equipo, por lo que en numerosas ocasiones tienen que parar el entrenamiento para corregir ejecuciones y dar explicaciones sobre los comportamientos técnico-tácticos a asimilar, reduciendo de esta forma la intensidad de la sesión y alejando los valores de RPE de los demandados por la competición. Además, las sesiones TAC fueron las de menor duración de las analizadas (Tabla 1), hecho que condiciona la menor RPE-TL alcanzada. Un aspecto clave para entender la menor duración de estas sesiones en nuestro estudio (Tabla 1) es el hecho de que la investigación se realizó completamente en pretemporada. Los jugadores suelen iniciar este período con un alto grado de desentrenamiento (Metaxas, Koutlianos, Sendelides \& Mandroukas, 2009), siendo necesario por tanto de un elevado volumen de entrenamiento para la mejora de la condición física en detrimento inicialmente del entrenamiento exclusivamente táctico.

Las sesiones con valores inferiores de intensidad y carga perceptiva de las analizadas fueron las ACTV, de forma similar a investigaciones previas (Campos-Vazquez et al., 2015). Estas sesiones, son realizadas el día previo a la disputa del partido. Durante la temporada competitiva, los entrenadores reducen de forma deliberada la carga de entrenamiento en estas sesiones (Impellizzeri et al., 2004; Malone et al., 2015), intentando descargar a los jugadores para facilitar su puesta a punto para el partido de competición. Los resultados obtenidos muestran como a pesar de las diferencias existentes entre el microciclo de pretemporada (con mayor carga y por tanto fatiga acumulada por los jugadores) y el de la temporada competitiva (Jeong et al., 2011), los entrenadores apuestan también por una reducción de la carga en el día previo a la disputa de partidos amistosos en pretemporada.

Debido a que ninguna de las sesiones analizadas consiguió simular los valores de intensidad y carga perceptiva reflejada en los partidos amistosos de pretemporada $(\mathrm{PA}+65)$, podría ser necesario aumentar el volumen y/o la exigencia de algunas sesiones de entrenamiento, para someter a los jugadores a una exigencia similar a la de la competición. No obstante, se desconoce si los valores de carga e intensidad habitualmente utilizados en las sesiones de entrenamiento planteadas, podrían ser suficientes para lograr las adaptaciones requeridas en pretemporada. Es bastante posible que el partido amistoso se presente como un estímulo de entrenamiento complementario a los diferentes tipos de sesión planteados, pudiendo resultar absolutamente necesario para que todos los jugadores de un mismo equipo puedan llegar al inicio del período competitivo con un nivel óptimo de condición física.

\section{Conclusiones}

De los resultados obtenidos en nuestra investigación, podemos extraer las siguientes conclusiones: los PA+65, reflejaron unos valores de RPE substancialmente superiores a los PA.45 (7.8 vs 6.8 u.a.), mostrando de esta forma que la RPE no puede ser considerada una medida de intensidad «pura». Además, ninguno de los tipos de sesión analizados (CF, TAC, ACTV), alcanzaron los niveles de intensidad y carga perceptiva reflejados por los PA+65. Por último, las sesiones de CF fueron el único tipo de sesión que alcanzaron valores de RPE (7.1 u.a.) y RPE-TL (597 u.a.) superiores a los mostrados por los PA.45 (6.8 y 509 u.a. respectivamente), mostrándose de esta forma como el único tipo de sesión que proporciona una carga perceptiva de entrenamiento superior a la disputa de 45 minutos de partido amistoso.

\section{Referencias}

Alexiou, H., \& Coutts, A. J. (2008). A comparison of methods used for quantifying internal training load in women soccer players. Int $J$ Sports Physiol Perform, 3(3), 320-330.

Anderson, L., Orme, P., Di Michele, R., Close, G. L., Morgans, R., Drust, B., \& Morton, J. P. (2016). Quantification of training load during one-, two- and three-game week schedules in professional soccer players from the English Premier League: implications for carbohydrate periodisation. J Sports Sci, 34(13), 1250-1259.

Arcos, A. L., Yanci, J., Mendiguchia, J., \& Gorostiaga, E. M. (2014). Rating of muscular and respiratory perceived exertion in professional soccer players. J Strength Cond Res, 28(11), 3280-3288.

Batterham, A. M., \& Hopkins, W. G. (2006). Making meaningful inferences about magnitudes. Int J Sports Physiol Perform, 1(1), 50-57

Borg, G, Hassmen, P., \& Lagerstrom, M. (1987). Perceived exertion related to heart rate and blood lactate during arm and leg exercise. Eur J Appl Physiol Occup Physiol, 56(6), 679-685.

Campos-Vazquez, M. A., Mendez-Villanueva, A., Gonzalez-Jurado, J.A., Leon-Prados, J. A., Santalla, A., \& Suarez-Arrones, L. (2015). Relationships between rating-of-perceived-exertion- and heart-ratederived internal training load in professional soccer players: a comparison of on-field integrated training sessions. Int J Sports Physiol Perform, 10(5), 587-592.

Clarke, N., Farthing, J. P., Norris, S. R., Arnold, B. E., \& Lanovaz, J. L. (2013). Quantification of training load in Canadian football: application of session-RPE in collision-based team sports. JStrength Cond Res, 27(8), 2198-2205.

Cummins, C., Orr, R. \& O’Connor, H. (2013). Global positioning systems (GPS) and microtechnology sensors in team sports: a systematic review. Sports Med, 43(10), 1025-42. 
Dellal, A. Drust, B. \& Lago-Penas, C., (2012). Variation of activity demands in small-sided soccer games. Int J Sports Med, 33, 370375.

Dellal, A., da Silva, C. D., Hill-Haas, S., Wong del, P., Natali, A. J., De Lima, J. R., . . Karim, C. (2012). Heart rate monitoring in soccer: interest and limits during competitive match play and training, practical application. J Strength Cond Res, 26(10), 2890-2906.

Di Salvo, V., Baron, R., Tschan, H., Calderon Montero, F. J., Bachl, N., \& Pigozzi, F. (2007). Performance characteristics according to playing position in elite soccer. Int J Sports Med, 28(3), 222-227.

Di Salvo, V., Gregson, W., Atkinson, G., Tordoff, P., \& Drust, B. (2009). Analysis of high intensity activity in Premier League soccer. Int $J$ Sports Med, 30(3), 205-212.

Foster, C., Florhaug, J. A., Franklin, J., Gottschall, L., Hrovatin, L. A., Parker, S., ... Dodge, C. (2001). A new approach to monitoring exercise training. J Strength Cond Res, 15(1), 109-115.

Hopkins, W. G. (2006). Spreadsheets for analysis of controlled trials, with adjustment for a subject characteristics. SportScience, 10, 4650

Impellizzeri, F. M., Rampinini, E., Coutts, A. J., Sassi, A., \& Marcora, S. M. (2004). Use of RPE-based training load in soccer. Med Sci Sports Exerc, 36(6), 1042-1047.

Jeong, T. S., Reilly, T., Morton, J., Bae, S. W., \& Drust, B. (2011). Quantification of the physiological loading of one week of «preseason» and one week of «in-season» training in professional soccer players. J Sports Sci, 29(11), 1161-1166.

Los Arcos, A., Gil-Rey, E., Izcue, I., \& Yanci, J. (2013). Monitoring training load in young professional soccer players. AGON Int $J$ Sport Sci, 3(1), 13-21.

Los Arcos, A., Mendez-Villanueva, A., Yanci, J., \& Martinez-Santos,
R. (2016). Respiratory and Muscular Perceived Exertion During Official Games in Professional Soccer Players. Int J Sports Physiol Perform, 11(3), 301-304.

Malone, J. J., Di Michele, R., Morgans, R., Burgess, D., Morton, J. P., \& Drust, B. (2015). Seasonal training-load quantification in elite English premier league soccer players. Int J Sports Physiol Perform, 10(4), 489-497.

Metaxas, T. I., Koutlianos, N., Sendelides, T., \& Mandroukas,A. (2009). Preseason physiological profile of soccer and basketball players in different divisions. J Strength Cond Res, 23(6), 1704-1713.

Murillo, V., Alvarez, J., \& Manomelles, P. (2016). Control de las cargas de entrenamiento a través de la percepción subjetiva. Predicción de la frecuencia cardíaca. Retos, 30( $2^{\circ}$ semestre), 82-86.

Owen, O., Djaoui, L., Newton, M., Malone, S., \& Mendes, B. (2017). A contemporary multi-modal mechanical approach to training monitoring in elite professional soccer. Science Med Football.Ahead of print.

Rampinini, E., Coutts, A. J., Castagna, C., Sassi, R., \& Impellizzeri, F. M. (2007). Variation in top level soccer match performance. Int $J$ Sports Med, 28(12), 1018-1024.

Sampaio, J., Abrantes, C., \& Leite, N. (2009). Power, heart rate and perceived exertion responses to $3 \times 3$ and $4 \times 4$ basketball smallsided games. Rev Psicol Dep, 18, 443-467.

Scott, B. R., Lockie, R. G., Davies, S. J. G., Clark, A. C., Lynch, D. M., \& de Jonge, X.A. K. (2014). The Physical demands of professional soccer players during in-season field-based training and matchplay. Journal of Australian Strength and Conditioning, 22(4), 7-15.

Wrigley, R., Drust, B., Stratton, G., Scott, M., \& Gregson, W. (2012). Quantification of the typical weekly in-season training load in elite junior soccer players. J Sports Sci, 30(15), 1573-1580.

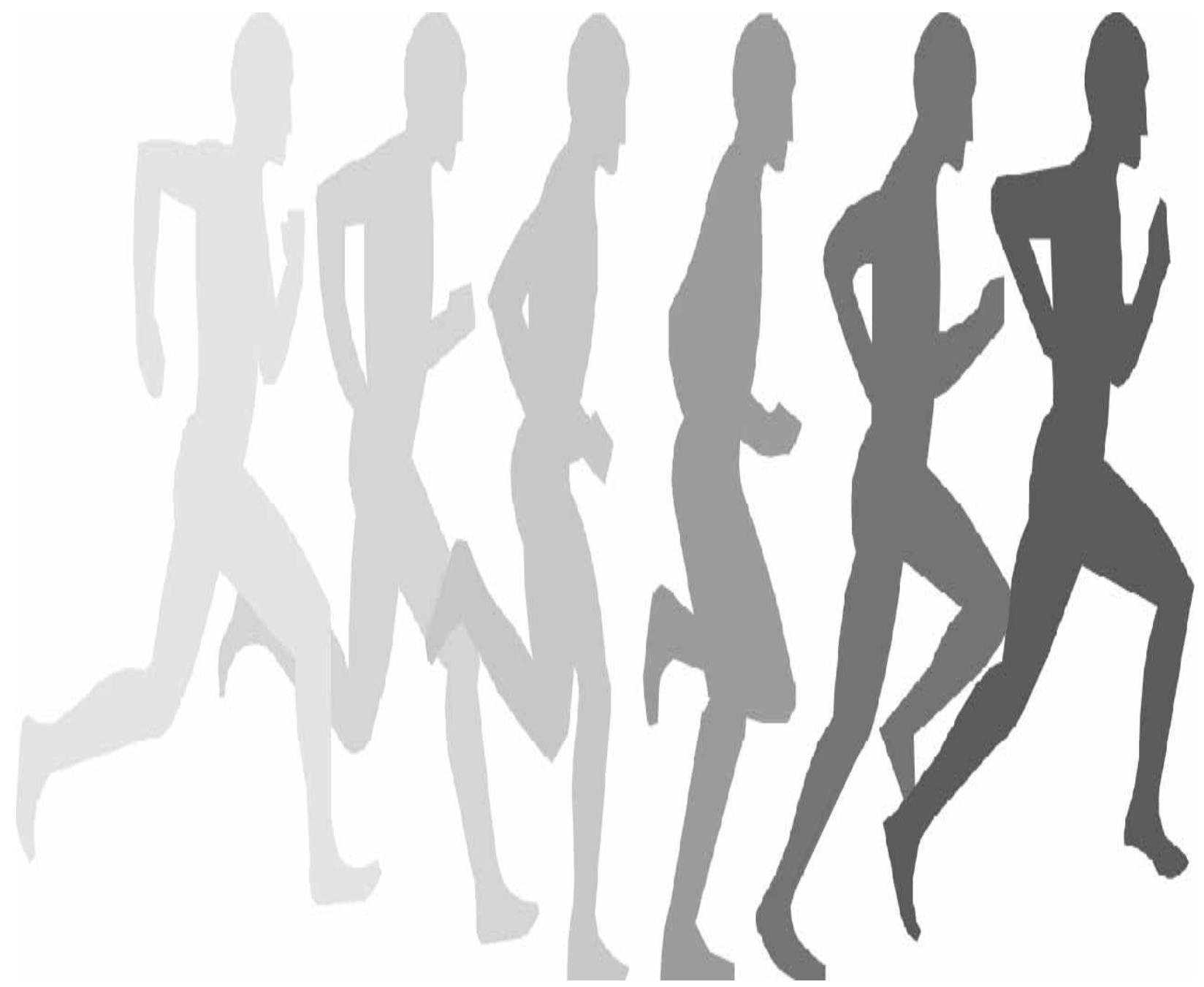

